\title{
Low-Complexity Robust Capon Beamforming Based on Reduced-Rank Technique
}

\author{
Zaifang Xi, ${ }^{1}$ Xiao-feng Wu, ${ }^{1}$ Shuyue $\mathrm{Wu}^{2}{ }^{2}$ Zhijun Tang, ${ }^{1}$ and Shigang $\mathrm{Hu}^{1}$ \\ ${ }^{1}$ School of Information and Electrical Engineering, Hunan University of Science and Technology, Xiangtan 411201, China \\ ${ }^{2}$ School of Information Science and Engineering, Hunan International Economics University, Changsha 410205, China \\ Correspondence should be addressed to Zaifang Xi; zfxi@hnust.edu.cn
}

Received 21 October 2014; Revised 28 December 2014; Accepted 30 December 2014

Academic Editor: Muhammad Taher Abuelma'atti

Copyright (c) 2015 Zaifang Xi et al. This is an open access article distributed under the Creative Commons Attribution License, which permits unrestricted use, distribution, and reproduction in any medium, provided the original work is properly cited.

\begin{abstract}
Existing robust Capon beamformers achieve robustness against steering vector errors at a high cost in terms of computational complexity. Computationally efficient robust Capon beamforming approach based on the reduced-rank technique is proposed in this paper. The proposed method projects the received data snapshots onto a lower dimensional subspace consisting of the matched filters of the multistage Wiener filter (MSWF). The subsequent adaptive beamforming will then be performed within this subspace. The combination of the benefit of the robust adaptive beamforming and the reduced-rank technique improves the performance on combating steering vector errors and lowering the computational complexity.
\end{abstract}

\section{Introduction}

The Capon beamformer chooses the weight vector by minimizing the array output power subject to a look direction constraint $[1,2]$. The standard Capon beamformer (SCB) has a high resolution and good interference suppression ability if the steering vector of the signal of interest (SOI) is known accurately [3]. However, the knowledge of the steering vector corresponding to the SOI may be imprecise because of some factors, such as DOA error, array calibration error, local scattering, near-far spatial signature mismatch, and finite sample effect [3-10]. Whenever this happens, the output SINR of the SCB degrades dramatically [6]. This effect is called signal selfnulling $[9,10]$.

Based on the uncertainty set of the steering vector, some robust beamformers were recently proposed [3, 4]. Using worst-case performance optimization, a novel method based on the second-order cone programming (SOCP) problem was proposed [4]. However, the SOCP problem has to rely on some specific optimization toolboxes such as $[11,12]$ to obtain its solution, which have a high computational cost and limit its practical implementation. The basic idea behind the robust Capon beamforming (RCB) approach of [3] is to estimate the desired steering vector in an uncertainty set by maximizing the array output power. The RCB approach needs to perform eigendecomposition on the sample covariance matrix, which also hits the wall of computational complexity.

In this paper, we devise a computationally efficient implementation of the RCB approach using the reduced-rank technique. A framework has been proposed for combining reduced-dimension and $\mathrm{RCB}$ methods, producing rapidly converging, low complexity reduced-dimension RCBs [13]. However, the author was not explicitly concerned with projection matrix design. Here, we propose to employ the matched filters of the MSWF as the projection matrix for reduced-rank processing. It can be proved that $D \geq P$ matched filters can form an orthogonal subspace containing the signal-plus-interference subspace, where $D$ and $P$ are the number of calculated matched filters of the MSWF and the number of signals, respectively. Thus, the matched filters of the MSWF can be used as the projection matrix. Moreover, the projected covariance matrix is tridiagonal, which can be directly calculated by means of the recursion procedure of the MSWF. Therefore, the proposed method has the advantage of computational simplicity to obtain its projection matrix and the projected covariance matrix. Furthermore, the matched 
filters are orthogonal with each other; thus the white noise property at the output of the projection preprocessor is preserved, thereby facilitating subsequent processing stages.

This paper is organized as follows. In Section 2, the signal model and a review of the RCB approach are given. The proposed method is introduced in Section 3. Simulation results are presented in Section 4 and conclusions are drawn in Section 5.

\section{Background}

2.1. Signal Model and Standard Capon Beamformer. Consider an $M$-sensor uniform linear array (ULA). The received data of the ULA at the $n$th snapshot can be expressed as

$$
\begin{aligned}
\mathbf{x}[n] & =\left[x_{1}[n], x_{2}[n], \ldots, x_{M}[n]\right]^{T} \\
& =\sum_{i=1}^{P} \mathbf{a}\left(\theta_{i}\right) s_{i}[n]+\mathbf{n}[n]
\end{aligned}
$$

where $x_{i}[n]$ is the received data at the $i$ th sensor, $[\cdot]^{T}$ denotes the transpose operation, $s_{i}[n]$ is the $i$ th source, the $M \times 1$ vector $\mathbf{n}[n]$ is the noise with a power $\sigma^{2}$, and

$$
\mathbf{a}\left(\theta_{i}\right)=\left[1, e^{-j 2 \pi d \sin \left(\theta_{i}\right) / \lambda}, \ldots, e^{-j 2 \pi(M-1) d \sin \left(\theta_{i}\right) / \lambda}\right]^{T}
$$

is the $M \times 1$ steering vector of the $i$ th signal in direction $\theta_{i}$, with $d$ being the adjacent sensor spacing and $\lambda$ denoting the signal wavelength.

Assume that all impinging signals and noise are uncorrelated with each other. Then the covariance matrix can be expressed as

$$
\mathbf{R}_{x x}=E\left[\mathbf{x}[n] \mathbf{x}[n]^{H}\right]=\sum_{i=1}^{P} \sigma_{i}^{2} \mathbf{a}\left(\theta_{i}\right) \mathbf{a}\left(\theta_{i}\right)^{H}+\sigma^{2} \mathbf{I},
$$

where $E[\cdot]$ denotes the expectation operation, $[\cdot]^{H}$ represents the Hermitian transpose, $\sigma_{i}^{2}$ is the $i$ th source power, and $\mathbf{I}$ is the $M \times M$ identity matrix. In practice, $\mathbf{R}_{x x}$ is replaced by the sample covariance matrix

$$
\widehat{\mathbf{R}}_{x x}=\frac{1}{N} \sum_{n=1}^{N} \mathbf{x}[n] \mathbf{x}^{H}[n],
$$

where $N$ is the number of snapshots.

Without loss of generality, we assume that the first signal is the SOI. Then the Capon beamformer is obtained by solving the following optimization problem:

$$
\begin{array}{ll}
\min _{\mathbf{w}} & \mathbf{w}^{H} \widehat{\mathbf{R}}_{x x} \mathbf{w} \\
\text { subject to } & \mathbf{w}^{H} \mathbf{a}\left(\bar{\theta}_{1}\right)=1,
\end{array}
$$

where $\mathbf{w}$ is the $M \times 1$ complex weight vector and $\bar{\theta}_{1}$ is the presumed steering direction.

The solution to (5) is given by

$$
\mathbf{w}=\frac{\widehat{\mathbf{R}}_{x x}^{-1} \mathbf{a}\left(\bar{\theta}_{1}\right)}{\mathbf{a}^{H}\left(\bar{\theta}_{1}\right) \widehat{\mathbf{R}}_{x x}^{-1} \mathbf{a}\left(\bar{\theta}_{1}\right)} .
$$

So the beamformer output power is given by

$$
P_{o}=\frac{1}{\mathbf{a}^{H}\left(\bar{\theta}_{1}\right) \widehat{\mathbf{R}}_{x x}^{-1} \mathbf{a}\left(\bar{\theta}_{1}\right)}
$$

2.2. RCB Approach. Based on the uncertainty set of the steering vector, the RCB approach can be formulated as follows [3]:

$$
\begin{array}{ll}
\min _{\mathbf{a}} & \mathbf{a}^{H} \widehat{\mathbf{R}}_{x x}^{-1} \mathbf{a} \\
\text { subject to } & \left\|\mathbf{a}-\mathbf{a}\left(\bar{\theta}_{1}\right)\right\|^{2} \leq \beta,
\end{array}
$$

where $\|\cdot\|$ denotes the Euclidean norm, a is the estimate of the desired steering vector, and $\beta$ is the uncertainty level. In order to prevent the solution $\mathbf{a}=0$, we assume that $\beta<\left\|\mathbf{a}\left(\bar{\theta}_{1}\right)\right\|^{2}$.

The problem (8) can be reformulated as the following quadratic problem with a quadratic equality constraint:

$$
\begin{array}{ll}
\min _{\mathbf{a}} & \mathbf{a}^{H} \widehat{\mathbf{R}}_{x x}^{-1} \mathbf{a} \\
\text { subject to } & \left\|\mathbf{a}-\mathbf{a}\left(\bar{\theta}_{1}\right)\right\|^{2}=\beta
\end{array}
$$

which can be solved using the Lagrange method. The solution is

$$
\mathbf{a}=\mathbf{a}\left(\bar{\theta}_{1}\right)-\left(\mathbf{I}+\lambda \widehat{\mathbf{R}}_{x x}\right)^{-1} \mathbf{a}\left(\bar{\theta}_{1}\right),
$$

where $\lambda$ is the Lagrange multiplier, which can be obtained as the solution to the spherical constraint equation

$$
g(\lambda)=\left\|\left(\mathbf{I}+\lambda \widehat{\mathbf{R}}_{x x}\right)^{-1} \mathbf{a}\left(\bar{\theta}_{1}\right)\right\|^{2}=\beta
$$

As noted in [3], the problem in (11) can be efficiently solved by applying a Newton-type iterative algorithm. Then the RCB weight vector is obtained by using (6) with $\mathbf{a}\left(\bar{\theta}_{1}\right)$ replaced by a.

\section{Proposed Method}

In this section, we first calculate the projection matrix using the matched filters of the MSWF, and then the adaptive reduced-rank beamforming is performed.

3.1. Calculating Projection Matrix. Let us define the reference signal and the observation data of the MSWF as $d_{0}[n]=x_{1}[n]$ and $\mathbf{x}_{0}[n]=\mathbf{x}[n]$, respectively. Let $\mathbf{h}_{m}$ and $\mathbf{B}_{m}$ denote the $m$ th matched filter and the $m$ th blocking matrix, respectively. The rank $D$ MSWF is given by the following set of recursions [14].

For $i=1, \ldots, D$ (forward recursion),

$$
\begin{gathered}
\mathbf{h}_{i}=\frac{E\left[\mathbf{x}_{i-1}[n] d_{i-1}^{*}[n]\right]}{\left\|E\left[\mathbf{x}_{i-1}[n] d_{i-1}^{*}[n]\right]\right\|} ; \\
d_{i}[n]=\mathbf{h}_{i}^{H} \mathbf{x}_{i-1}[n] ; \\
\mathbf{B}_{i}=\mathbf{I}-\mathbf{h}_{i} \mathbf{h}_{i}^{H} ; \\
\mathbf{x}_{i}[n]=\mathbf{B}_{i}^{H} \mathbf{x}_{i-1}[n] .
\end{gathered}
$$


Decrement $i=D, \ldots, 1$ (backward recursion):

$$
\begin{gathered}
w_{i}=\frac{E\left[d_{i-1}^{*}[n] e_{i}[n]\right]}{E\left[\left|e_{i}[n]\right|^{2}\right]} ; \\
e_{i-1}[n]=d_{i-1}[n]-w_{i}^{*} e_{i}[n],
\end{gathered}
$$

where $e_{D}[n]=d_{D}[n]$.

We now form a matrix $\mathbf{H}$, given by

$$
\begin{aligned}
\mathbf{H} & =\left[\mathbf{h}_{1}, \mathbf{B}_{1} \mathbf{h}_{2}, \ldots, \prod_{i=1}^{D-1} \mathbf{B}_{i} \mathbf{h}_{D}\right] \\
& =\left[\mathbf{h}_{1}, \mathbf{h}_{2}, \ldots, \mathbf{h}_{D}\right] .
\end{aligned}
$$

It is shown in [15] that the matrix $\mathbf{H}$ forms an orthogonal basis for the Krylov subspace; namely,

$$
\operatorname{span}\left\{\mathbf{h}_{1}, \mathbf{h}_{2}, \ldots, \mathbf{h}_{D}\right\}=\operatorname{span}\left\{\mathbf{r}, \mathbf{R}_{x x} \mathbf{r}, \ldots, \mathbf{R}_{x x}^{D-1} \mathbf{r}\right\},
$$

where $\mathbf{r}=E\left[\mathbf{x}_{0}[n] d_{0}^{*}[n]\right]$ is the cross-correlation between the reference signal and the observation data. Since all impinging signals and noise are uncorrelated with each other, $\mathbf{r}$ can also be written as

$$
\mathbf{r}=\sum_{i=1}^{P} \sigma_{i}^{2} \mathbf{a}\left(\theta_{i}\right)+\sigma^{2} \mathbf{e},
$$

where $\mathbf{e}=[1,0, \ldots, 0]^{T}$. It is easily shown that, for any $k>0$, $\mathbf{R}_{x x}^{k} \mathbf{r}$ contains all the array steering vectors $\mathbf{a}\left(\theta_{i}\right)(i=1, \ldots$, $P)$. Thus, the signal-plus-interference subspace is contained in $\mathbf{H}$ if $D \geq P$. Hence, an orthogonal basis

$$
\mathbf{H}=\left[\mathbf{h}_{1}, \ldots, \mathbf{h}_{D}\right] \quad(D \geq P)
$$

can be employed as the projection matrix for projecting the full-dimension received snapshots onto a lower dimensional subspace, and the adaptive beamforming then occurs within this subspace.

3.2. Performing Adaptive Beamforming. Using (17) as the projection matrix, the reduced-rank beamforming problem can be formulated as follows:

$$
\begin{array}{ll}
\min _{\mathbf{w}_{r}} & \mathbf{w}_{r}^{H} \widehat{\mathbf{R}}_{r} \mathbf{w}_{r} \\
\text { subject to } & \mathbf{w}_{r}^{H} \mathbf{a}_{r}\left(\bar{\theta}_{1}\right)=1,
\end{array}
$$

where $\mathbf{w}_{r}$ is the $D \times 1$ complex reduced-rank weight vector and $\widehat{\mathbf{R}}_{r}=\mathbf{H}^{H} \widehat{\mathbf{R}}_{x x} \mathbf{H}$ and $\mathbf{a}_{r}\left(\bar{\theta}_{1}\right)=\mathbf{H}^{H} \mathbf{a}\left(\bar{\theta}_{1}\right)$ are the projected sample covariance matrix and the projected presumed steering vector, respectively. It should be noted that $\mathbf{H}^{H} \widehat{\mathbf{R}}_{x x} \mathbf{H}$ is tridiagonal, which can be directly calculated by applying the recursion procedure of the MSWF (see [14] for details), avoiding estimating the full-rank sample covariance matrix defined in (4). The solution to (18) is given by

$$
\mathbf{w}_{r}=\frac{\widehat{\mathbf{R}}_{r}^{-1} \mathbf{a}_{r}\left(\bar{\theta}_{1}\right)}{\mathbf{a}_{r}\left(\bar{\theta}_{1}\right)^{H} \widehat{\mathbf{R}}_{r}^{-1} \mathbf{a}_{r}\left(\bar{\theta}_{1}\right)} .
$$

Then the output power is

$$
P_{r}=\frac{1}{\mathbf{a}_{r}\left(\bar{\theta}_{1}\right)^{H} \widehat{\mathbf{R}}_{r}^{-1} \mathbf{a}_{r}\left(\bar{\theta}_{1}\right)} .
$$

Following the classic RCB approach, the proposed reduced-rank RCB approach can be expressed as

$$
\begin{array}{ll}
\min _{\mathbf{a}_{r}} & \mathbf{a}_{r}^{H} \widehat{\mathbf{R}}_{r}^{-1} \mathbf{a}_{r} \\
\text { subject to } & \left\|\mathbf{a}_{r}-\mathbf{a}_{r}\left(\bar{\theta}_{1}\right)\right\|^{2}=\beta_{r},
\end{array}
$$

where $\beta_{r}$ denotes the uncertainty level for the reduced-rank RCB and $\mathbf{a}_{r}$ is the estimate of the projected desired steering vector, given by

$$
\mathbf{a}_{r}=\mathbf{a}_{r}\left(\bar{\theta}_{1}\right)-\left(\mathbf{I}+\eta \widehat{\mathbf{R}}_{r}\right)^{-1} \mathbf{a}_{r}\left(\bar{\theta}_{1}\right)
$$

where $\eta$ is the Lagrange multiplier, which can be obtained by solving the following problem:

$$
g(\eta)=\left\|\left(\mathbf{I}+\eta \widehat{\mathbf{R}}_{r}\right)^{-1} \mathbf{a}_{r}\left(\bar{\theta}_{1}\right)\right\|^{2}=\beta_{r}
$$

Similarly, the solution to (23) can be obtained using the same Newton-type iterative algorithm as in [3]. The reduced-rank weight vector is then obtained using (19) with $\mathbf{a}_{r}\left(\theta_{1}\right)$ replaced by $\mathbf{a}_{r}$. Finally, the weight vector of the proposed approach is expressed as

$$
\mathbf{w}=\mathbf{H} \frac{\widehat{\mathbf{R}}_{r}^{-1} \mathbf{a}_{r}}{\mathbf{a}_{r}^{H} \widehat{\mathbf{R}}_{r}^{-1} \mathbf{a}_{r}} .
$$

3.3. Selection of $\beta_{r}$. In this section, the selection of $\beta_{r}$ is investigated. It has been shown in [16] that the uncertainty level $\beta$ for the traditional RCB method should be chosen as small as possible such that

$$
\beta \geq \min _{\gamma}\left\|\overline{\mathbf{a}} e^{j \gamma}-\mathbf{a}\left(\bar{\theta}_{1}\right)\right\|^{2}
$$

where $\overline{\mathbf{a}}$ is the actual steering vector. Therefore, for the proposed method, we choose the value of $\beta_{r}$ as

$$
\beta_{r} \geq \min _{\gamma}\left\|\overline{\mathbf{a}}_{r} e^{j \gamma}-\mathbf{a}_{r}\left(\bar{\theta}_{1}\right)\right\|^{2}
$$

where $\overline{\mathbf{a}}_{r}=\mathbf{H}^{H} \overline{\mathbf{a}}$ is the projected actual steering vector. However, the actual steering vector is an unknown vector. Thus, we assume that the SOI comes from a DOA uncertainty region with a high probability, while no interference comes from this region. It should be noted that this assumption is also implemented in the beamformers of $[7,17,18]$. Suppose that the uncertainty region is defined as $\Phi=\left[\bar{\theta}_{1}-\Delta \theta, \bar{\theta}_{1}+\Delta \theta\right]$, where $\Delta \theta$ is the DOA uncertainty range [18]. Therefore, the values 
of $\beta$ and $\beta_{r}$ for the traditional RCB method and the proposed method, respectively, can be defined as

$$
\begin{gathered}
\beta \geq \max \left(\min _{\gamma}\left\|\mathbf{a}\left(\bar{\theta}_{1}-\Delta \theta\right) e^{j \gamma}-\mathbf{a}\left(\bar{\theta}_{1}\right)\right\|^{2},\right. \\
\left.\min _{\gamma}\left\|\mathbf{a}\left(\bar{\theta}_{1}+\Delta \theta\right) e^{j \gamma}-\mathbf{a}\left(\bar{\theta}_{1}\right)\right\|^{2}\right), \\
\beta_{r} \geq \max \left(\min _{\gamma}\left\|\mathbf{a}_{r}\left(\bar{\theta}_{1}-\Delta \theta\right) e^{j \gamma}-\mathbf{a}_{r}\left(\bar{\theta}_{1}\right)\right\|^{2},\right. \\
\left.\min _{\gamma}\left\|\mathbf{a}_{r}\left(\bar{\theta}_{1}+\Delta \theta\right) e^{j \gamma}-\mathbf{a}_{r}\left(\bar{\theta}_{1}\right)\right\|^{2}\right) .
\end{gathered}
$$

3.4. Computational Complexity. To estimate the sample covariance matrix, a computational complexity of $O\left(M^{2} N\right)$ is needed. The eigendecomposition operation needs a computational complexity of $O\left(M^{3}\right)$. Thus the full-rank RCB approach requires a complexity of $O\left(M^{3}\right)+O\left(M^{2} N\right)$. The SOCP-based methods of $[4,5]$ have at least complexity of $O\left(M^{3.5}\right)+$ $\mathrm{O}\left(\mathrm{M}^{2} \mathrm{~N}\right)$, and the SOCP-based methods of $[7,8]$ that attempt to further improve the robustness with respect to the methods of $[3,4]$ have much more computational complexity. The dominant computational cost of the proposed method is the calculation of the matched filters and the reduced-rank weight vector. The calculation for each matched filter needs around a computational complexity of $O(M N)$. To calculate the reduced-rank weight vector, a computational complexity of $O\left(D^{3}\right)$ is required. Thus, the proposed approach needs a computational complexity of $O(D M N)+O\left(D^{3}\right)$. Consequently, the proposed approach has a lower computational cost than the existing robust beamforming methods.

\section{Simulations}

In this section, simulations are carried out to investigate the performance of the proposed method compared with the SCB and the RCB. Since the signal subspace based methods will not work if the signal-plus-interference subspace is underestimated, that is, $D<P$, we only consider the cases of $D \geq P$. We consider a ULA with $M=10$ sensors and half-wavelength spacing between adjacent sensors. The SOI arrives from direction $\theta_{1}=0^{\circ}$. Two interfering signals with interference-to-noise ratio (INR) of $30 \mathrm{~dB}$ impinge on the array from the directions $-40^{\circ}$ and $50^{\circ}$, respectively. The array is steered toward the direction $\bar{\theta}_{1}=\theta_{1}+\Delta_{1}$, where $\Delta_{1}$ is the DOA mismatch. Here, both the gain and phase errors are considered. In this case, the actual steering vector can be written as $\overline{\mathbf{a}}(\theta)=\Gamma \mathbf{a}(\theta)$, where $\Gamma=\operatorname{diag}\left[\alpha_{1} e^{-j \psi_{1}}, \ldots, \alpha_{M} e^{-j \psi_{M}}\right]$ is the diagonal matrix of the calibration errors, with $\alpha_{k}$ and $\psi_{k}$ standing for the amplitude and phase errors, respectively. We assume that the amplitude and phase errors have a uniform distribution: $\alpha_{k} \in[0.8,1.2]$ and $\psi_{k} \in[-\pi / 100, \pi / 100]$. Note that $\Gamma$ changes from run to run while remaining constant for all snapshots. The DOA uncertainty range is set to $\Delta \theta=4^{\circ}$. The uncertainty level $\beta$ for the traditional RCB method and the value of $\beta_{r}$ for the proposed method are calculated using (27) and (28), respectively. All results are averaged based on 100 independent simulation runs.

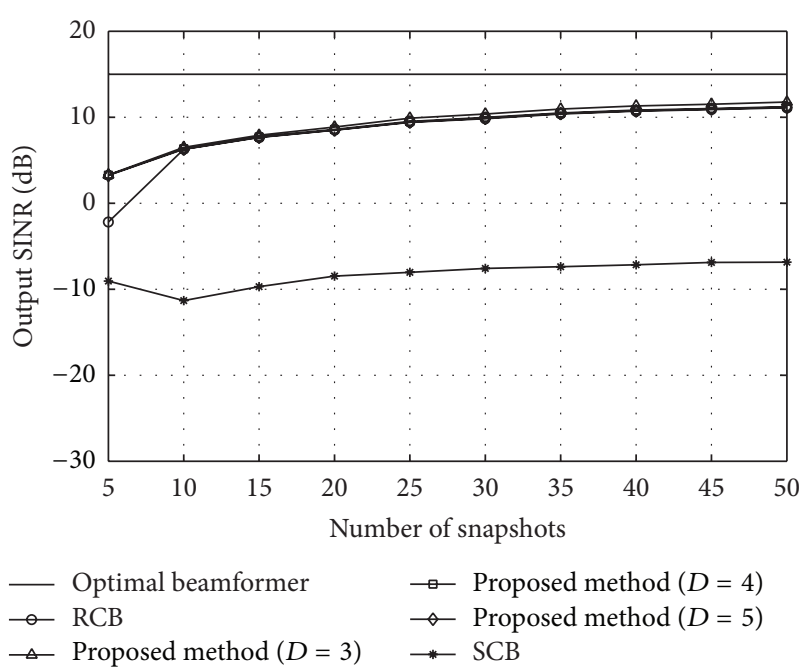

FIGURE 1: Output SINR versus the number of snapshots.

4.1. Output SINR versus the Number of Snapshots. In the first example, we consider the effect of the number of snapshots on the output SINR of the beamformers. The input signalto-noise ratio (SNR) of the SOI is set to $5 \mathrm{~dB}$ and the DOA mismatch is $\Delta_{1}=2^{\circ}$. Figure 1 shows the output SINR of the beamformers versus the number of snapshots. As shown, the output SINR of the SCB degrades significantly with a DOA mismatch of $2^{\circ}$; however, both the proposed method and the traditional RCB approach can provide sufficient robustness against steering vector errors. Moreover, the proposed method can achieve a fast convergence rate due to the reduced-rank processing, leading to a much better performance than the traditional RCB approach when the number of snapshots is very small. Additionally, it can be seen that the proposed method can still achieve a good performance when the rank $D$ is larger than $P$.

4.2. Output SINR versus SNR. In the second example, we investigate the effect of the input SNR on the performance of the beamformers. The number of snapshots is fixed at $N=50$. Other parameters remain the same as in the first example. Figure 2 shows the output SINR of the beamformers as a function of the input SNR. It can be clearly seen from this figure that the proposed method has a good performance in the high SNR region; however, the performance of the proposed method of $D=3$ is much worse than that of the other methods considered in the low SNR region due to the problem of possible subspace swap.

4.3. Output SINR versus DOA Mismatch. In the third example, the DOA mismatch is uniformly distributed on $\left[0,6^{\circ}\right]$ while the actual DOA of the SOI is $0^{\circ}$. The number of snapshots is $N=50$ and other parameters remain the same as in the first example. The result of output SINR versus DOA mismatch is shown in Figure 3. It can be observed that when the DOA mismatch increases, the performance of the traditional RCB approach degrades dramatically; however, the proposed method still achieves a higher output SINR than 


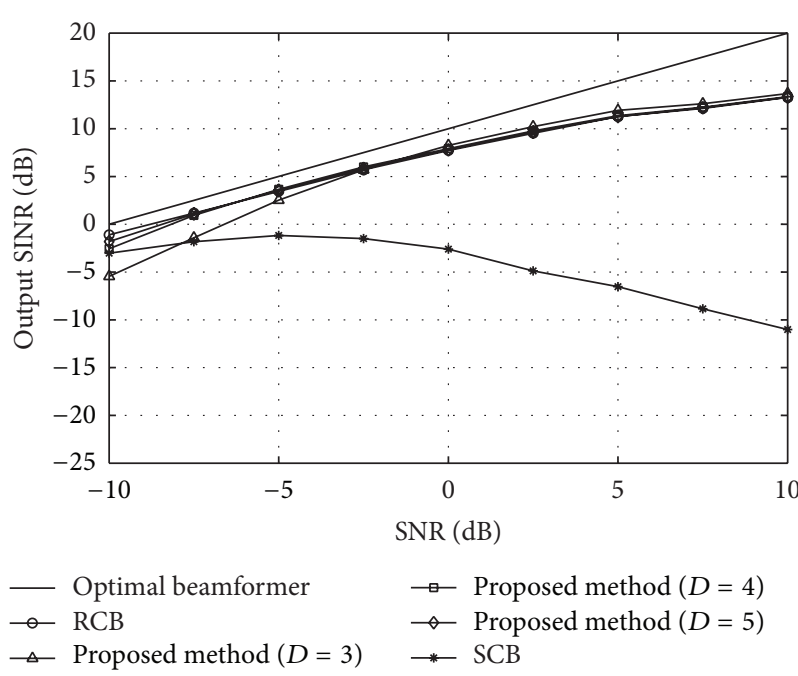

FIGURE 2: Output SINR of the beamformers versus input SNR.

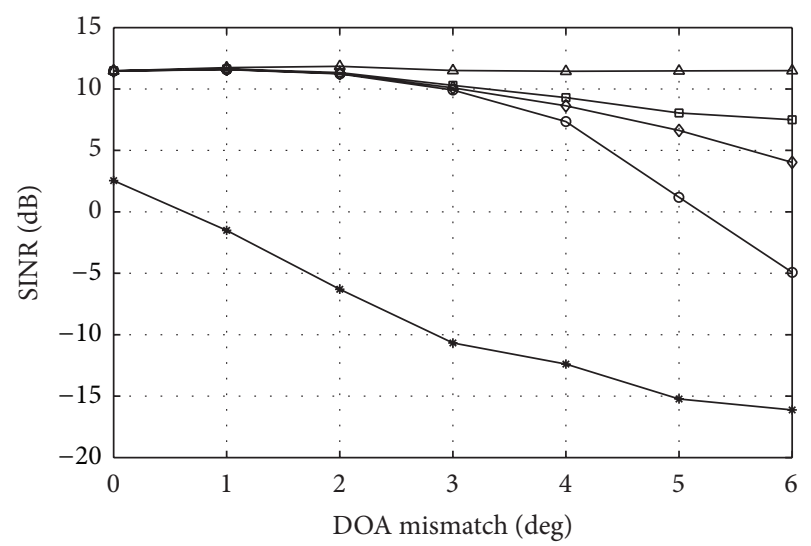

$\begin{array}{ll}\longrightarrow \text { Optimal beamformer } & \rightarrow \text { Proposed method }(D=4) \\ \rightarrow \text { RCB } & \rightarrow \text { Proposed method }(D=5) \\ \triangle \text { Proposed method }(D=3) & \rightarrow \text { - SCB }\end{array}$

FIGURE 3: Output SINR of the beamformers versus DOA mismatch.

the traditional RCB approach. In addition, as the rank $D$ increases the performance of the proposed method degrades. This is because more noise components are included in the projection matrix which, in turn, degrades its ability of accommodating the increased DOA mismatch.

\section{Conclusions}

A low complexity RCB approach based on reduced-rank technique has been proposed for improving the robustness of the SCB against steering vector errors. Unlike the traditional full-rank RCB approach, the proposed method performs the adaptive beamforming within a lower dimensional subspace that consists of the matched filters of the MSWF, thereby reducing its computational complexity and the finite-sample effect. Simulation results have been presented to demonstrate the effectiveness of the proposed method.

\section{Conflict of Interests}

The authors declare that there is no conflict of interests regarding the publication of this paper.

\section{Acknowledgments}

The authors are grateful to the two anonymous reviewers for their very useful suggestions and comments. This study was supported by the National Nature Science Foundation of China (nos. 61274026, 61376076, and 61377024), supported by the Science and Technology Plan Foundation of Hunan Province (nos. 2013FJ2011, 2014FJ2017) and supported by the Scientific Research Fund of Hunan Provincial Education Department (nos. 14A084, 14B060).

\section{References}

[1] J. Capon, "High-resolution frequency-wavenumber spectrum analysis," Proceedings of the IEEE, vol. 8, no. 57, pp. 1408-1418, 1969.

[2] H. L. van Trees, Optimum Array Processing, Part IV of Detection, Estimation, and Modultion Theory, Wiley, New York, NY, USA, 2002.

[3] J. Li, P. Stoica, and Z. S. Wang, "On robust Capon beamforming and diagonal loading," IEEE Transactions on Signal Processing, vol. 51, no. 7, pp. 1702-1715, 2003.

[4] S. A. Vorobyov, A. B. Gershman, and Z. Q. Luo, "Robust adaptive beamforming using worst-case performance optimization: a solution to the signal mismatch problem," IEEE Transactions on Signal Processing, vol. 51, no. 2, pp. 313-324, 2003.

[5] S. A. Vorobyov, H. Chen, and A. B. Gershman, "On the relationship between robust minimum variance beamformers with probabilistic and worst-case distortionless response constraints," IEEE Transactions on Signal Processing, vol. 56, no. 11, pp. 5719-5724, 2008.

[6] J. Li and P. Stoica, Eds., Robust Adaptive Beamformer, Wiley, New York, NY, USA, 2005.

[7] Z. L. Yu, W. Ser, M. H. Er, Z. H. Gu, and Y. Q. Li, "Robust adaptive beamformers based on worst-case optimization and constraints on magnitude response," IEEE Transactions on Signal Processing, vol. 57, no. 7, pp. 2615-2628, 2009.

[8] S. E. Nai, W. Ser, Z. L. Yu, and S. Rahardja, "A robust adaptive beamforming framework with beampattern shaping constraints," IEEE Transactions on Antennas and Propagation, vol. 57, no. 7, pp. 2198-2203, 2009.

[9] W. Zhang, J. Wang, and S. Wu, "Robust minimum variance multiple-input multiple-output radar beamformer," IET Signal Processing, vol. 7, no. 9, pp. 854-862, 2013.

[10] W. Zhang, J. Wang, and S. Wu, "Adaptive multiple-input mu ltiple-output radar beamforming based on direct data domain approach," IET Radar, Sonar and Navigation, vol. 6, no. 8, pp. 632-638, 2014.

[11] J. F. Sturm, "Using SeDuMi 1.02, a MATLAB toolbox for optimization over symmetric cones," Optimization Methods \& Software, vol. 11, no. 1-4, pp. 625-653, 1999.

[12] M. Grant and S. Boyd, CVX: Matlab Software for Disciplined Convex Programming, 2008, http://www.standford.eud/boyd/ crx. 
[13] S. D. Somasundaram, "Reduced dimension robust Capon beamforming for large aperture passive sonar arrays," IET Radar, Sonar and Navigation, vol. 5, no. 7, pp. 707-715, 2011.

[14] J. S. Goldstein, I. S. Reed, and L. L. Scharf, "A multistage representation of the Wiener filter based on orthogonal projections," IEEE Transactions on Information Theory, vol. 44, no. 7, pp. 2943-2959, 1998.

[15] M. L. Honig and W. Xiao, "Performance of reduced-rank linear interference suppression," IEEE Transactions on Information Theory, vol. 47, no. 5, pp. 1928-1946, 2001.

[16] J. Li, P. Stoica, and Z. Wang, "Doubly constrained robust Capon beamformer," IEEE Transactions on Signal Processing, vol. 52, no. 9, pp. 2407-2423, 2004.

[17] W. Zhang, S. Wu, and J. Wang, "Robust Capon beamforming in presence of large DOA mismatch," Electronics Letters, vol. 49, no. 1, pp. 75-76, 2013.

[18] W. Zhang, J. Wang, and S. Wu, "Robust Capon beamforming against large DOA mismatch," Signal Processing, vol. 93, no. 4, pp. 804-810, 2013. 

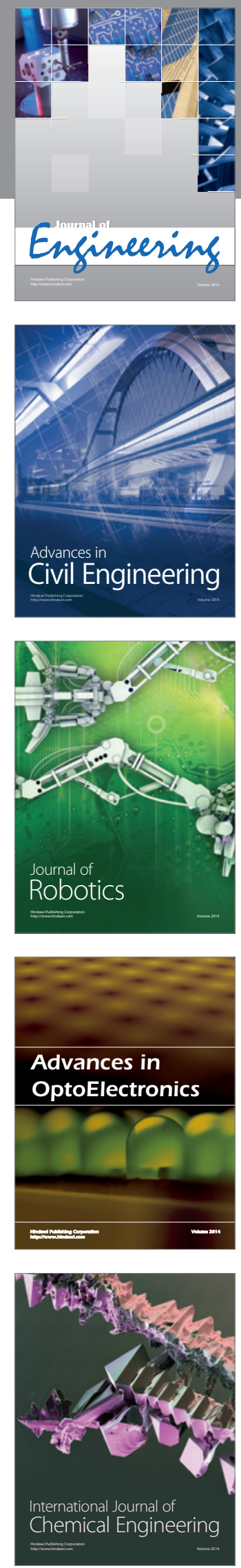

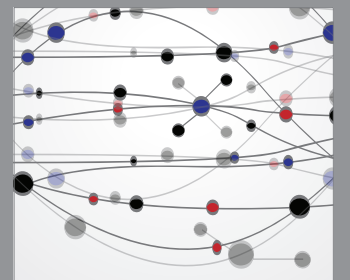

The Scientific World Journal
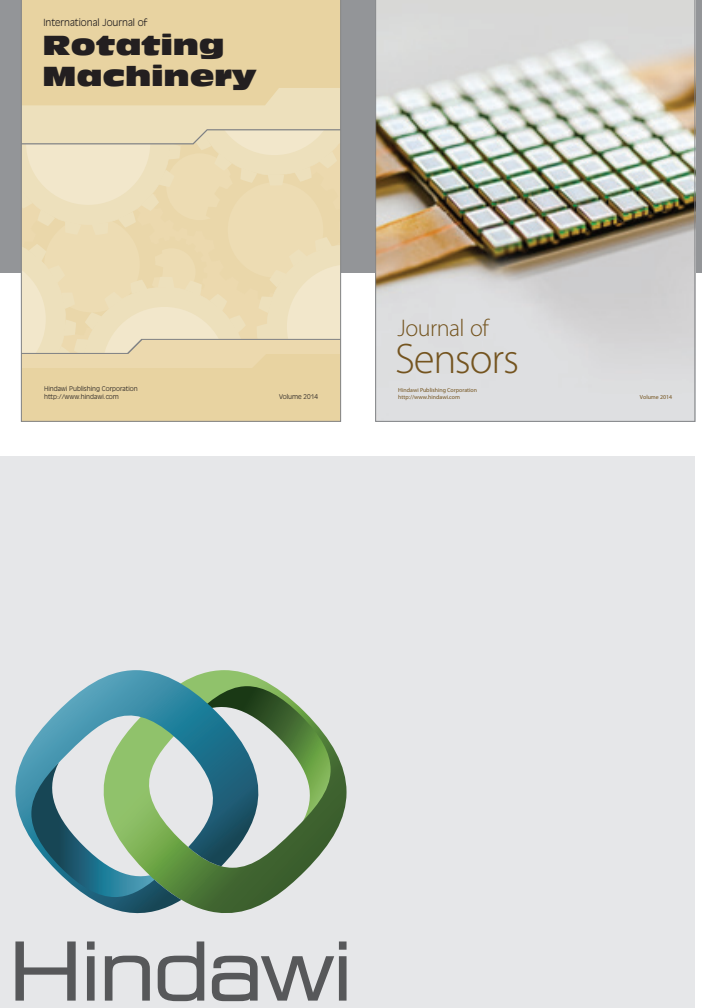

Submit your manuscripts at http://www.hindawi.com
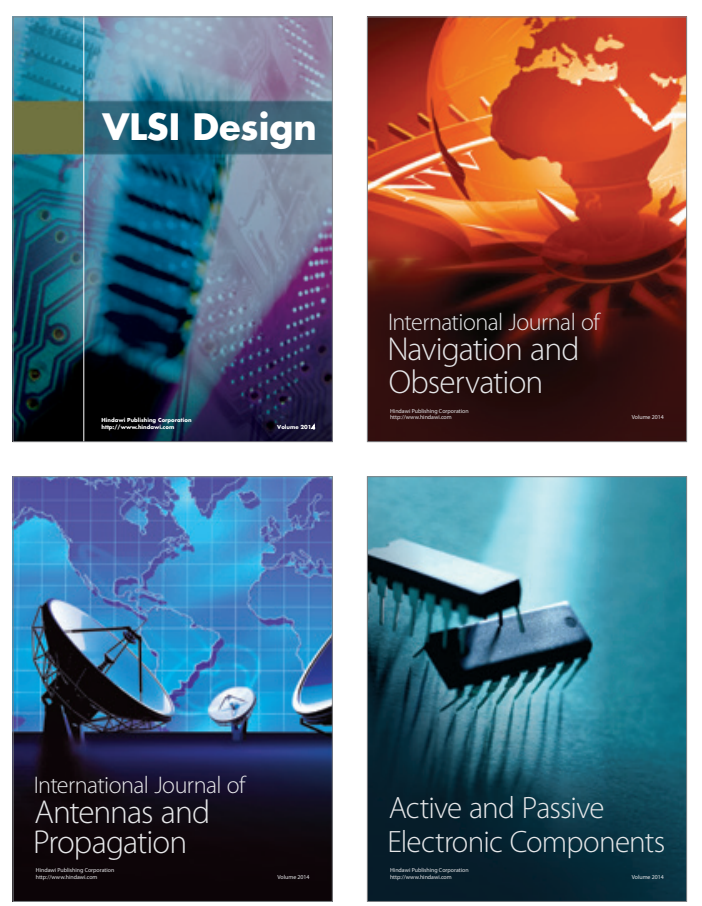
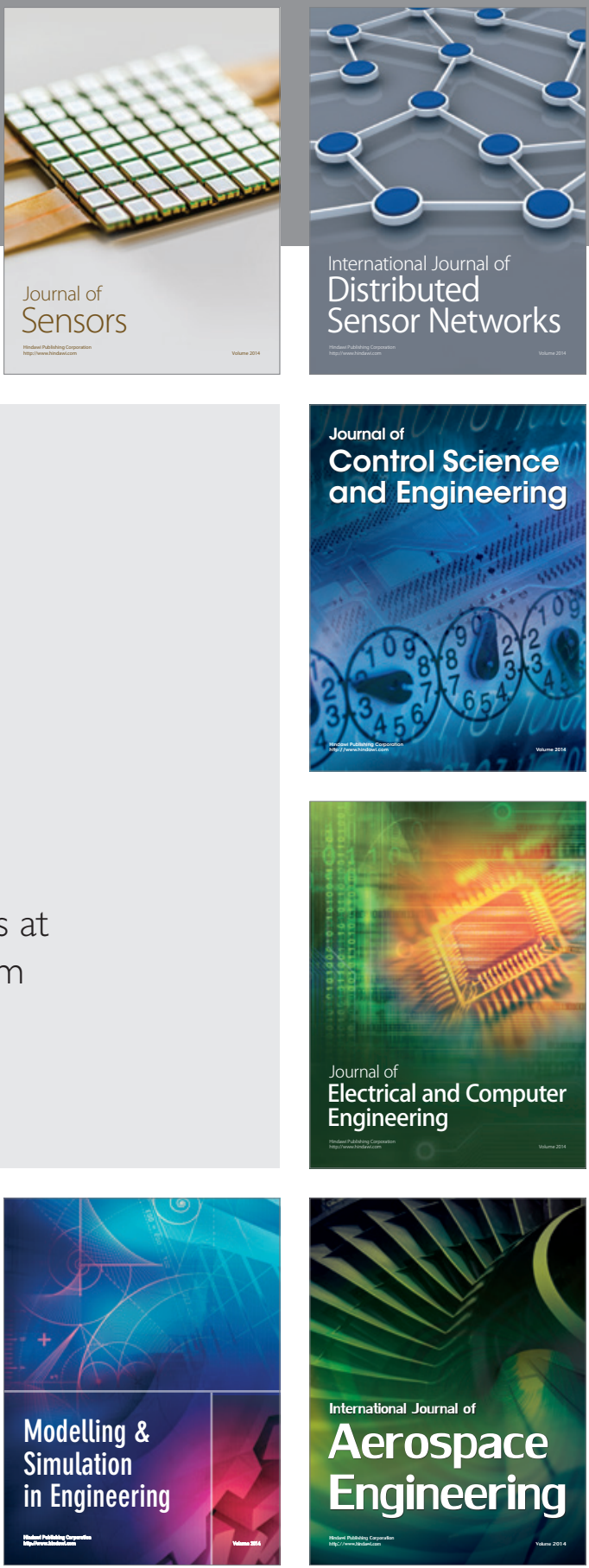

Journal of

Control Science

and Engineering
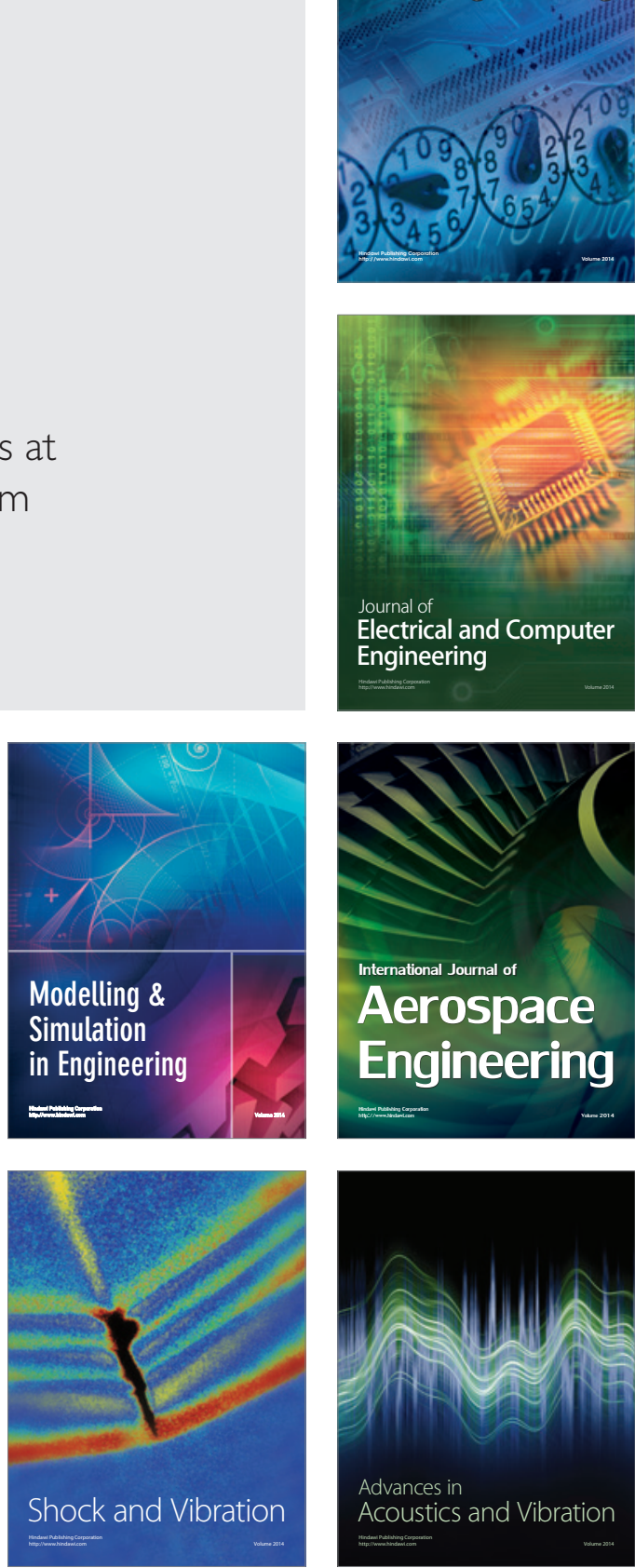